\title{
HADAMARD'S THREE CIRCLES THEOREM
}

\author{
RAPHAEL M. ROBINSON
}

Hadamard's theorem is concerned with the relation between the maximum absolute values of an analytic function on three concentric circles. ${ }^{1}$ If we put

$$
M(r)=\max _{|z|=r}|f(z)|,
$$

then the theorem states that $\log M(r)$ is a convex function of $\log r$ for $r^{\prime}<r<r^{\prime \prime}$, if $f(z)$ is regular for $r^{\prime}<|z|<r^{\prime \prime}$. This is an immediate consequence of the fact that if $|f(z)| \leqq A|z|^{\lambda}$ on two circles about the origin, then it is also true between the circles; and this in turn is seen by applying the principle of maximum to $f(z) / z^{\lambda}$. The bound is attainable within the ring only for $f(z)=\alpha z^{\lambda}$ with $|\alpha|=A$. Notice that this function is single-valued only if $\lambda$ is an integer, so that Hadamard's bound is not in general sharp for single-valued functions. (It is the sharp bound for the class of many-valued functions, any branch of which is regular in the ring, and for which $|f(z)|$ is singlevalued.)

We shall consider only single-valued functions. The problem of finding the sharp bound in Hadamard's theorem is formulated as Problem A below. (It is no essential restriction to suppose that the radius of the outer circle is 1 , and that the given bound on this circle is 1.) Problems $B$ and $C$ raise the same question for more special classes of functions.

Problem A. Suppose $0<q<Q<1$ and $p>0$. Consider the class of functions satisfying the following conditions: $f(z)$ is regular for $q \leqq|z| \leqq 1$,

$$
|f(z)| \leqq 1 \text { for }|z|=1, \quad|f(z)| \leqq p \quad \text { for }|z|=q .
$$

An address delivered before the Berkeley meeting of the Society on April 29, 1944, by invitation of the Program Committee; received by the editors May 6, 1944.

1 The theorem was stated (without proof) in Hadamard's note, Sur les fonctions entières, Bull. Soc. Math. France vol. 24 (1896) pp. 186-187. His proof was apparently first published in 1912; it may be found in footnote 2, p. 94, of Selecta: Jubile Scientifique de M. Jacques Hadamard, Paris, 1935. In the meantime, proofs (of a less simple nature) had been given by $O$. Blumenthal and by G. Faber. See Blumenthal, Über ganze transzendente. Funktionen, Jber. Deutschen Math. Verein. vol. 16 (1907) pp. 97109 , and Sur le mode de croissance des fonctions entières, Bull. Soc. Math. France vol. 35 (1907) pp. 213-232; Faber, Über das Anwachsen analytischer Funktionen, Math. Ann. vol. 63 (1907) pp. 549-551. 
Let $P$ be the largest value of $|f(Q)|$ for any function of the given class. How much is $P$, and for what functions is it attained?

Problem B. The same as Problem A, with the additional hypothesis that the coefficients of the Laurent series for $f(z)$ are positive or zero.

Problem C. The same as Problem A, with the additional hypotheses that $p<1$ and that $f(z)$ is regular also for $|z|<q$ (hence for $|z| \leqq 1$ ).

Remarks. From any function $f(z)$ with $|f(Q)|=P$ we can obtain a function $H(z)$ with $H(Q)=P$, by putting $H(z)=P f(z) / f(Q)$. The name extremal function will be applied only to an admissible function with $H(Q)=P$.

We may determine a real $\lambda$ such that $q^{\lambda}=p$. If $\lambda$ is an integer, then $H(z)=z^{\lambda}$ is the extremal function for all three problems; but if $\lambda$ is not an integer, then $z^{\lambda}$ is not an admissible function. We may restrict ourselves to the latter case, and shall use $n$ to denote the integer such that $n-1<\lambda<n$.

If we indicate the dependence of the extremal function on $p$ by using the notation $H(z, p)$, then it is clear that for the first two problems we have

$$
H\left(z, p q^{k}\right)=z^{k} H(z, p)
$$

for any integer $k$, since a power of $z$ times an admissible function is admissible; but for Problem $\mathrm{C}$ no such relation is to be expected. Consequently, there is no loss of generality in supposing $q<p<1$ (that is, $n=1$ ) when studying Problems A and B.

Summary of results. ${ }^{2}$ We state here some of the principal results that are known concerning the three problems. For each of the problems, the extremal function $H(z)$ exists and is unique, and is real for real $z$. It is univalent if $q<p<1$. In Problems A and $\mathrm{B}, H(z)$ is independent of $Q$; and the same is true in Problem $\mathrm{C}$ at least if $q<p<1$. We tabulate some additional results in the three cases for comparison.

Problem A.

$z H^{\prime}(z) / H(z)$ is an elliptic function of $\log z$.

2 Problem A was first solved by 0 . Teichmüller, Eine Verschärfung des Dreikreisesatzes, Deutsche Mathematik vol. 4 (1939) pp. 16-22. But we shall follow here the solution given by the author in Analytic functions in circular rings, Duke Math. J. vol. 10 (1943) pp. 341-354. Problem B was solved by F. Carlson, Sur le module maximum d'une fonction analytique uniforme, Arkiv för Mathematik, Astronomi, och Fysik vol. 26A (1938). Problem C is studied by M. H. Heins in a paper, On a problem of Walsh concerning the Hadamard three circles theorem, Trans. Amer. Math. Soc. vol. 55 (1944) pp. 349-372, which I had the privilege of reading before publication. 
$\mid \begin{aligned} & H(z) \mid=1 \text { for }|z|=1 \\ & H(z) \mid=p \text { for }|z|=q .\end{aligned}$

If $q<p<1, w=H(z)$ maps $q<|z|<1$ on $|w|<1$ omitting an arc of $|w|=p$.

Problem B.

$H(z)$ is an average of $z^{n-1}$ and $z^{n}$.

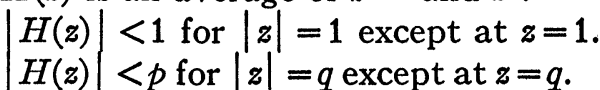

If $q<p<1, w=H(z)$ is a contraction with 1 as fixed point.

Problem C.

$H(z)$ is a rational function of the $n$th degree.

$\left|\begin{array}{l}H(z) \\ H(z)\end{array}\right|<p$ for $|z| z \mid=1$.

If $q<p<1, w=H(z)$ maps the unit circle onto itself.

Study of Problem A. We suppose $q<p<1$. It may be seen from general mapping theorems that there exists a function $H(z)$ which maps the ring $q<|z|<1$ on $|w|<1$ omitting an arc of $|w|=p$. We may suppose that $p$ is the midpoint of the omitted arc. The function $H(z)$ is regular on the boundaries of the ring, and we have

$$
|H(z)|=1 \text { for }|z|=1, \quad|H(z)|=p \quad \text { for }|z|=q .
$$

If $f(z)$ is any admissible function, then $|f(z) / H(z)| \leqq 1$ on both boundaries of the ring. We could apply the principle of maximum to conclude that $|f(Q) / H(Q)| \leqq 1$, were it not for the fact that $H(z)$ has a zero in the ring, so that $f(z) / H(z)$ has a pole. The fundamental lemma of the author's paper provides an extension of this principle which enables the conclusion to be drawn nevertheless. The lemma states that if a function is regular in a circular ring except for one simple pole, and does not exceed 1 in absolute value on the boundaries, then it is less than 1 on the radius opposite the pole. Applying this lemma, we verify that $H(z)$ is the desired extremal function.

By applying Schwarz's reflection principle, we can continue $H(z)$ to the whole plane excluding 0 and $\infty$. The reflections on the outer and inner boundaries give the relations

$$
H(1 / z)=1 / H(z), \quad H\left(q^{2} / z\right)=p^{2} / H(z),
$$

if we use the fact that $H(z)$ is real for real $z$. From these it follows that

$$
H\left(q^{2} z\right)=p^{2} H(z),
$$

and hence 


$$
q^{2} z B^{\prime}\left(q^{2} z\right) / H\left(q^{2} z\right)=z H^{\prime}(z) / H(z) .
$$

Thus $z H^{\prime}(z) / H(z)$ is an elliptic function of $\log z$ with the periods $2 \log q$ and $2 \pi i$.

It is not difficult to obtain the explicit formula

$$
H(z)=z \theta(q z / p) / \theta(p z / q)
$$

where

$$
\theta(z)=\sum_{k=-\infty}^{\infty} q^{k^{2}} z^{k} .
$$

This enables easy calculation of the extremal function.

Study of Problem B. Here

$$
f(z)=\sum_{k=-\infty}^{\infty} c_{k} z^{k}
$$

with $c_{k} \geqq 0$, hence $M(r)=f(r)$. Evidently

$$
M^{\prime \prime}(r) \geqq 0,
$$

the equality holding only if $M(r)=c_{0}+c_{1} r$. If $q<p<1$, we can determine positive $c_{0}$ and $c_{1}$ so that $M(1)=1, M(q)=p$; that is, so that

$$
c_{0}+c_{1}=1, \quad c_{0}+c_{1} q=p .
$$

With this determination of $c_{0}$ and $c_{1}$, the extremal function is

$$
H(z)=c_{0}+c_{1} z \text {. }
$$

This result of Carlson, which concerns a special class of functions, has an interesting application to the more general class previously considered. In fact, if we no longer suppose that $f(z)$ has positive coefficients, we have nevertheless that the average of $|f(z)|^{2}$ on $|z|=r$ is

$$
\sum_{k=-\infty}^{\infty}\left|c_{k}\right|^{2} r^{2 k}
$$

which is a power series in $r^{2}$ with positive coefficients, so that Carlson's result may be applied. If we suppose given that the average of $|f(z)|^{2}$ on $|z|=1$ does not exceed 1 , and that the average on $|z|=q$ does not exceed $p^{2}(q<p<1)$, then the function $H(z)$ having the largest quadratic mean on $|z|=Q$ is of the form

$$
H(z)=c_{0}+c_{1} z
$$


where $c_{0}$ and $c_{1}$ are subject to the conditions

$$
\left|c_{0}\right|^{2}+\left|c_{1}\right|^{2}=1, \quad\left|c_{0}\right|^{2}+\left|c_{1}\right|^{2} q^{2}=p^{2} .
$$

Some lemmas. We consider now some results that are used in the study of Problem C. The results concern functions constant in absolute value on a circle, and interpolation by bounded functions.

In the first place, an equality such as $|F(z)|=1$ either holds identically on $|z|=1$ or at but a finite number of points, provided $F(z)$ is regular on $|z|=1$. For on the circle, $|F(z)|=1$ is equivalent to

$$
F(z) \bar{F}(1 / z)=1 \text {. }
$$

Since the left side is regular on the circle, the result follows.

Suppose now that $F(z)$ is regular for $|z| \leqq 1$ and that $|F(z)|=1$ for $|z|=1$. Let $a_{1}, a_{2}, \cdots, a_{n}$ be the zeros of $F(z)$ in $|z|<1$. Then we find that

$$
F(z)=\alpha \prod_{k=1}^{n} \frac{z-a_{k}}{1-\bar{a}_{k} z} \quad(|\alpha|=1)
$$

by applying the principle of maximum and minimum to $F(z)$ divided by the product on the right. The zeros and poles of $F(z)$ are inverse with respect to the given circle $|z|=1$. A similar result holds for any other circle. If $|F(z)|$ were constant on two circles about the origin, $F(z)$ being regular within the larger circle, then the zeros and poles would have to be inverse with respect to both circles, that is, the zeros at 0 and the poles at $\infty$, and hence $F(z)=\alpha z^{n}$.

Concerning interpolation by bounded functions, we need the following theorem. Let $z_{1}, z_{2}, \cdots, z_{n}, \zeta$ be $n+1$ distinct points in $|z|<1$, and let $w_{1}, w_{2}, \cdots, w_{n}, \omega$ be any $n+1$ points in $|w| \leqq 1$. Consider the class of functions $F(z)$ regular for $|z|<1$ and with $|F(z)| \leqq 1$ there. The number of such functions satisfying the interpolating conditions

$$
F\left(z_{k}\right)=w_{k} \quad(k=1,2, \cdots, n)
$$

may be 0,1 , or $\infty$. If there is just one such function, then it is rational of less than the nth degree, and satisfies $|F(z)|=1$ for $|z|=1$. If there are infinitely many such functions, then the possible values of $F(\zeta)$ fill a closed circle; the additional condition

$$
F(\zeta)=\omega
$$

will determine the function uniquely if and only if $\omega$ is on the boundary of that circle.

The proof is by induction. Consider first the case $n=0$. The num- 
ber of functions is infinite. The possible values of $F(\zeta)$ fill the circle $|w| \leqq 1$. The condition $F(\zeta)=\omega$ determines $F(z)$ uniquely, if and only if $|\omega|=1$.

Suppose now that $n>0$. In case $\left|w_{n}\right|=1$, if there is any solution it is $F(z)=w_{n}$, which is a rational function of the zeroth degree and satisfies $|F(z)|=1$ for $|z|=1$. In case $\left|w_{n}\right|<1$, we establish a one-toone correspondence between the given functions and those satisfying certain interpolating conditions at the points $z_{1}, \cdots, z_{n-1}$ by means of the equation

$$
G(z)=\frac{F(z)-w_{n}}{1-\bar{w}_{n} F(z)}: \frac{z-z_{n}}{1-\bar{z}_{n} z} .
$$

The various desired conclusions about $F(z)$ follow easily from the corresponding conclusions about $G(z)$.

Study of Problem C. Here the case $q<p<1$ has a very simple solution (but the general case cannot be reduced to it). For if the condition $|f(z)| \leqq p$ for $|z|=q$ is replaced by the weaker condition $|f(q)|$ $\leqq p$, then it follows (using Schwarz's lemma) that the maximum possible value of $|f(Q)|$ is attained by the linear function $H(z)$ which maps the unit circle onto itself, with \pm 1 as fixed points, and $H(q)=p$. But this function clearly satisfies $|H(z)| \leqq p$ for $|z|=q$ (under the hypothesis $q<p<1$ ), and hence is the required extremal function.

For the general case, Heins reaches his results by a rather indirect method. It will be convenient to modify the statement of Problem C by supposing that $f(z)$ is regular for $|z|<1$ rather than for $|z| \leqq 1$. The condition $|f(z)| \leqq 1$ for $|z|=1$ may be replaced by $|f(z)|<1$ for $|z|<1$. With this modification, the existence of an extremal function $H(z)$ is clear from the theory of normal families. It will be shown later that this function is unique, regular for $|z|=1$, and that $|H(z)|=1$ for $|z|=1$.

As a first step, we show that if $H(z)$ is regular for $|z|=1$, then $|H(z)|=1$ there. Otherwise, $|H(z)|=1$ at only a finite number of points on $|z|=1$, and hence we can find a small $\operatorname{arc} A B$ of $|z|=1$, near 1 , where $|H(z)|<1$. Choose $K>1$ so that $K|H(z)|<1$ on this arc. Now it is easy to construct a function $g(z)$, regular for $|z| \leqq 1$ except at $A$ and $B$, with constant absolute values on every circular arc joining $A$ and $B$, these values varying from $K$ on the given $\operatorname{arc} A B$ to 1 on the complementary arc of the unit circle. Since $|H(z) g(z)| \leqq 1$ for $|z|=1$, except at $A$ and $B$, and $|H(z) g(z)|<1$ near these points, it follows that $|H(z) g(z)|<1$ for $|z|<1$. The function $H(z) g(z)$ may fail to be an admissible $f(z)$ by being too large on $|z|=q$. However, 
$g(z)$ is larger at $Q$ than on $|z|=q$, so that if we divide $H(z) g(z)$ by the maximum of $g(z)$ on $|z|=q$, we have a function $f(z)$ which is admissible and for which $|f(Q)|>|H(Q)|$, which is impossible.

Next we see that $|H(z)|=p$ has only a finite number of roots on $|z|=q$. For otherwise it would be an identity, and $H(z)$ would be rational. But then $H(z)$ would also be regular for $|z|=1$, and hence $|H(z)|=1$ there. From the fact that $|H(z)|$ is constant on two circles, we should conclude that $H(z)=z^{n}$, and hence $p=q^{n}$, which is the case we have excluded.

We shall now show that in the hypothesis that $|f(z)| \leqq p$ for $|z|=q$, only a finite number of points on $|z|=q$ have any weight. Let $H(z)$ be an extremal function, and let $z_{1}, z_{2}, \cdots, z_{l}$ be the points on $|z|=q$ where $|H(z)|=p$. Then if $|F(z)|<1$ for $|z|<1$, and $\left|F\left(z_{k}\right)\right| \leqq p(k=1,2, \cdots, l)$, we can conclude that $|F(Q)| \leqq P$ (that is, we obtain the same bound for $|F(Q)|$ as if we had supposed that $|F(z)| \leqq p$ for $|z|=q)$. For if there were such a function with $|F(Q)|>P$, then we could also find a function with $\left|F\left(z_{k}\right)\right|<p$ and $F(Q)>P$. But then we see that

$$
f(z)=(1-\epsilon) H(z)+\epsilon F(z)
$$

is admissible (if $\epsilon$ is sufficiently small), and that $f(Q)>P$.

The next step is to see that $H(z)$ can be determined by interpolation. We shall show that if $|F(z)|<1$ for $|z|<1$, if $F\left(z_{k}\right)=H\left(z_{k}\right)$ $(k=1,2, \cdots, l)$, and if $F(Q)=H(Q)$, then $F(z)=H(z)$ identically. Consider first the interpolating problem defined by $F\left(z_{k}\right)=H\left(z_{k}\right)$. The possible values of $F(Q)$ are restricted to a circle including $P$. If $P$ were not on the boundary, then $F(Q)>P$ would be possible. Thus $P$ is on the boundary, and the additional condition $F(Q)=P$ serves to determine $F(z)$ uniquely.

Thus $H(z)$ is a rational function of at most the $l$ th degree, with $|H(z)|=1$ for $|z|=1$. From this the uniqueness of $H(z)$ follows at once. For if both $H_{1}(z)$ and $H_{2}(z)$ were extremal, then so also would be their average. This average must also satisfy the condition $|H(z)|=1$ for $|z|=1$, which is possible only if $H_{1}(z)=H_{2}(z)$ on $|z|=1$ and hence identically. Furthermore, if $H(z)$ is extremal, so also is $\bar{H}(z)$; hence $\bar{H}(z)=H(z)$, or $H(z)$ is real for real $z$.

Now consider the degree of $H(z)$. In the first place, $|H(z)|=p$ has $l$ different roots on $|z|=q$, and these are all of even order, since $|H(z)| \leqq p$ on $|z|=q$. In other words, the equation

$$
H(z) H\left(q^{2} / z\right)=p^{2}
$$

has at least $2 l$ roots, so that $H(z)$ cannot be of less than the $l$ th de- 
gree. Thus $H(z)$ is exactly of the $l$ th degree, and the roots of the displayed equation all lie on $|z|=q$, and are double roots.

As in the introduction, let $n$ be the integer such that $n-1<\lambda<n$. We shall give a sketch of the proof that $l=n$.

In the first place, it is not difficult to show that $P$ is a continuous function of $q, p, Q$; and using this fact, it may be shown that (for $|z|<1) H(z)$ depends continuously on these parameters.

Next we notice that $l$ is a function of $n$ only. For as long as we exclude the case in which $\lambda$ is an integer, $l$ is the number of double roots of $H(z) H\left(q^{2} / z\right)-p^{2}$ on $|z|=q$. Since this function is regular and depends continuously on the parameters for $q^{2}<|z|<1$, and has roots only on $|z|=q$, it cannot gain or lose a root.

Recalling that the degree of $H(z)$ is equal to the number of zeros in $|z|<1$, it is easy to see that the degree is a lower semi-continuous function of the parameters. Since the degree is $n$ when $\lambda=n$, it cannot be less than $n$ when $\lambda$ is slightly less than $n$. Combined with the preceding result, this shows that $l \geqq n$.

Finally, by an ingenious method which we cannot consider here, Heins finds (for any given $n$ ) some cases in which it can be shown that the degree of $H(z)$ does not exceed $n$. This completes the proof that $H(z)$ is of exactly the $n$th degree when $n-1<\lambda<n$, and that $|H(z)|=p$ has exactly $n$ different roots on $|z|=q$.

University of CALIFornia 